\title{
Measurement of Acceptable Noise Level with Background Music
}

\author{
Hyun-Jung Ahn ${ }^{1,2}$, Junghwa Bahng ${ }^{2}$, and Jae Hee Lee ${ }^{2}$ \\ 1Department of Inter-Disciplinary Program Molecular Medicine, Hallym University College of Medicine, Anyang, \\ ${ }^{2}$ Department of Audiology, Hallym University of Graduate Studies, Seoul, Korea
}

\begin{abstract}
Received January 3, 2015
Revised March 11, 2015

Accepted April 29, 2015
\end{abstract}

\author{
Address for correspondence \\ Jae Hee Lee, PhD \\ Department of Audiology, \\ Hallym University of \\ Graduate Studies, \\ 405 Yeoksam-ro, Gangnam-gu, \\ Seoul 135-841, Korea \\ Tel $+82-2-2051-2942$ \\ Fax +82-2-3453-6618 \\ E-mail1eejaehee@hallym.ac.kr
}

\begin{abstract}
Background and Objectives: Acceptable noise level (ANL) is a measure of the maximum background noise level (BNL) that a person is willing to tolerate while following a target story. Although researchers have used various sources of target sound in ANL measures, a limited type of background noise has been used. Extending the previous study of Gordon-Hickey \& Moore (2007), the current study determined the effect of music genre and tempo on ANLs as possible factors affecting ANLs. We also investigated the relationships between individual ANLs and the familiarity of music samples and between music ANLs and subjective preference. Subjects and Methods: Forty-one participants were seperated into two groups according to their ANLs, 29 low-ANL listeners and 12 high-ANL listeners. Using Korean ANL material, the individual ANLs were measured based on the listeners' most comfortable listening level and BNL. The ANLs were measured in six conditions, with different music tempo (fast, slow) and genre (K-pop, pop, classical) in a counterbalanced order. Results: Overall, ANLs did not differ by the tempo of background music, but music genre significantly affected individual ANLs. We observed relatively higher ANLs with K-pop music and relatively lower ANLs with classical music. This tendency was similar in both low-ANL and high-ANL groups. However, the subjective ratings of music familiarity and preference affected ANLs differently for low-ANL and high-ANL groups. In contrast to the low-ANL listeners, the ANLs of the high-ANL listeners were significantly affected by music familiarity and preference. Conclusions: The genre of background music affected ANLs obtained using background music. The degree of music familiarity and preference appears to be associated with individual susceptibility to background music only for listeners who are greatly annoyed by background noise (high-ANL listeners).

J Audiol Otol 2015;19(2):79-84
\end{abstract}

KEY WORDS: Acceptable noise level · Background music · Familiarity · Preference.

\section{Introduction}

Background noise is pervasive in a wide range of our everyday environments including home, workplace, school, transportation, store, and recreational spaces. Under noisy environments, some people may experience difficulties understanding speech in noise whereas some may have less problems. Considering the large variability in individual susceptibility to

This is an Open Access article distributed under the terms of the Creative Commons Attribution Non-Commercial License (http://creativecommons. org/licenses/by-nc/3.0/) which permits unrestricted non-commercial use, distribution, and reproduction in any medium, provided the original work is properly cited. noise, Nabelek, et al. [1] quantified the lowest signal to noise ratio that a person could accept while listening to speech. This noise level that an individual can tolerate or accept is called acceptable noise level (ANL). The ANL test appears to be a quick, reliable, clinician-friendly procedure [2]. Unlike speech-in-noise recognition test, listener does not need to repeat the target speech they heard for ANL measurement. Rather, the ANL is calculated by difference between the most comfortable level for speech and the maximum level of background noise that the listener is willing to accept while following the target story.

Although various types of sound soruces have been used as target sound such as reversed, unfamiliar discourses, and non- 
semantic sound sources [3-5], little research has focused the individual susceptibility to interference from background music [6-8]. To our knowlege, only a few studies $[1,6]$ have reported ANLs to background music using different genres of music. In the previous study of Nabelek, et al. [1], 'light music' of waiting room was first used as source of background noise to examine listeners' ANLs to music. In Gordon-Hickey and Moore [6], music of rock genre was only used as background noise for ANL measurement. Comparing the results of young normal-hearing adults between two studies, Nabelek and colleagues [1] reported that 'light music' was more demanding compared to multi-talker babble noise whereas Gordon-Hickey and Moore [6] found more acceptance of rockgenre music than babble noise. For the inconsistent finding, the authors speculated a substantial impact of musical attributes such as music tempo, genre, familiarity, and musical experience.

Since no studies have examined the influence of music tempo and genre on ANLs, the present study aimed to determine whether music tempo and genre would affect individual ANLs. In addition, this study investigated whether music familiarity and preference would influence the ANLs. To facilitate comparison with the previous finding of Gordon-Hickey and Moore [6], this study grouped participants into low-ANL group (listeners with greater acceptance of noise) and high-ANL group (listeners with lower acceptance of noise). This would allow us to investigate whether music preference or familiarity would affect ANLs differently between low-ANL and high-ANL groups.

\section{Subjects and Methods}

\section{Participants}

A total of forty-one (21 males, 20 females) native Korean listeners participated in this study. Accoridng to the previous criteria [6,9], the participants consisted of 29 low-ANL listeners $(\leq 6 \mathrm{~dB})$ and 12 high-ANL listeners $(\geq 14 \mathrm{~dB})$. All the participants had Type-A tympanogram with no history of neurologic pathology. The mean age of low-ANL group $(n=29)$ was 47.2 years of age [standard deviation (SD): 23.4], and the mean age of high-ANL group $(n=12)$ was 47.1 years of age (SD: 17.0). The mean puretone threshold of two groups was less than $20 \mathrm{~dB}$ HL from 250 to $2000 \mathrm{~Hz}$, and less than $30 \mathrm{~dB}$ $\mathrm{HL}$ at 4000 and $6000 \mathrm{~Hz}$. Analyses of independent t-test showed that the ages and the octave-scale puretone thresholds from 250 to $6000 \mathrm{~Hz}$ did not significantly differ ( $p>0.05$ for all frequencies) between low-ANL and high-ANL groups. Additional analyses of Pearson correlation verified no significant relations between ANLs and puretone threshold average and also between ANLs and ages of individuals, consistent with previous finding [1]. Before the experimental test, participants were informed of anticipated risk and benefit and all were agreed to participation in research before the experimental testing.

\section{Stimuli and procedure}

For the ANL measure, a recorded discourse of a male talker in Korean [10] was used as target story. As background noise, ANLs were tested in six different music categories consisting of three music genres (Korean-pop music: K-pop, popular music: pop, and classical music: classical) and two types of music tempo (fast and slow). Each of the six categories included three music samples, resulting in 18 music samples as total, as displayed in Table 1. Selecting as either fast or slow tempo was based on two aspects as follows. First, as an objective standard, the number of beats per minute (BPM) was considered, as in the previous criteria [11]. Thus, 94 or more BPM was required to be fast tempo music whereas 72 or less BPM for the slow tempo music. Especially for the classical music, the tempo was based on the Quarter-notes Per Minute (QPM) such that Adagio (60-80 QPM) classical music was chosen as slow classical music and Allegro (120-160 QPM) was selected as fast classical music. Second, as described in the previous studies which measured ANLs to background music [1,6], a single instrumental part was avoided such that vocal parts with lyrics were selected as music samples of K-pop and pop genres. In contrast, the instrumental part without lyrics was selected as music sample of classical genre. All the wavefiles were downloaded from K-pop archive charts. The average root mean square value of each wavefile was equated via Adobe Audition version 3.0 (Adobe Systems Incorporated, San Jose, CA, USA) with 16-bit resolution and a sampling rate of $44.1 \mathrm{kHz}$. The order for the presentation of background music was randomly selected for each participant.

All the stimuli were delivered via clinical audiometer (Madsen Obiter 922, GN Otometrics, Taastrup, Denmark) connected to an external CD player (DEJ017, Sony, Tokyo, Japan) and a loudspeaker at 0 degrees azimuth to the subject. Prior to the experimental test, a practice with verbal and written instructions in Korean [10] was given to ensure whether the participant understood task correctly. Subjects' ANLs were obtained based on the procedure of the previous study [1] which first introduced ANL. For the ANL measure, each participant was required to adjust level of a target story in $2 \mathrm{~dB}$ steps to find individual's most comfortable level (MCL). While listening to and following the target message at MCL, the listener was then required to adjust level of background music in $2 \mathrm{~dB}$ steps to find the maximum level that the listener was willing to accept, called background noise level (BNL). The ANL 
Table 1. Music samples used as background noise

\begin{tabular}{|c|c|c|c|c|}
\hline Genre & Tempo & Title of music & Artist & Year \\
\hline \multirow[t]{6}{*}{ K-pop } & Slow & Happy me & Eco & 1997 \\
\hline & Slow & I know only love & Subong Sim & 1987 \\
\hline & Slow & You'll never know & Euni Hye & 1975 \\
\hline & Fast & Festival & Junghwa Uhm & 1999 \\
\hline & Fast & Round and round & $\mathrm{Na}-\mathrm{Mi}$ & 1984 \\
\hline & Fast & Like Indian Doll & $\mathrm{Na}-\mathrm{Mi}$ & 1990 \\
\hline \multirow[t]{6}{*}{ Pop } & Slow & Like a star & Corinne Bailey Rae & 2006 \\
\hline & Slow & When I dream & Crystal Gayle & 1978 \\
\hline & Slow & The rose & Bette Midler & 1979 \\
\hline & Fast & Single ladies & Beyonce & 2011 \\
\hline & Fast & Sunny & Boney M & 1976 \\
\hline & Fast & Conga & Glpria Estefan & 1985 \\
\hline \multirow[t]{6}{*}{ Classical } & Slow & $\begin{array}{l}\text { Sonata for Flute and Basso Continuo No. } 2 \\
\text { in E minor BWV } 1034\end{array}$ & Johann Sebastian Bach & 1720 \\
\hline & Slow & Flute Concerto in G major, K.313/285c & Mozart, Wolfgang Amadeus & 1777 \\
\hline & Slow & Air on a G String & Johann Sebastian Bach & 1717 \\
\hline & Fast & $\begin{array}{l}\text { Beeethoven symphonie Nr.9. Adagio } \\
\text { molto e cantabile }\end{array}$ & Ludwig van Beethoven & 1824 \\
\hline & Fast & Four seasons, Summer & Antonio Vivaldi & 1725 \\
\hline & Fast & L'estro armonico & Antonio Vivaldi & 1711 \\
\hline
\end{tabular}

K-pop: Korean-pop music, Pop: popular music, Classical: classical music

Table 2. Mean ANL values (standard deviations) of low-ANL and high-ANL groups depending on music tempo and genre

\begin{tabular}{|c|c|c|c|c|c|c|c|c|c|c|}
\hline & \multicolumn{3}{|c|}{ Slow music } & \multicolumn{3}{|c|}{ Fast music } & \multirow{2}{*}{$\begin{array}{l}\text { K-pop } \\
\text { mean }\end{array}$} & \multirow{2}{*}{$\begin{array}{l}\text { Pop } \\
\text { mean }\end{array}$} & \multirow{2}{*}{$\begin{array}{c}\text { Classical } \\
\text { mean }\end{array}$} & \multirow{2}{*}{$\begin{array}{l}\text { Total } \\
\text { means }\end{array}$} \\
\hline & K-pop & Pop & Classical & K-pop & Pop & Classical & & & & \\
\hline Low-ANL group & $2.5(2.0)$ & $2.2(2.2)$ & $2.3(2.4)$ & $4.1(2.3)$ & $3.0(1.9)$ & $3.1(2.4)$ & $3.3(2.0)$ & $2.6(1.9)$ & $2.7(2.2)$ & $2.9(1.8)$ \\
\hline High-ANL group & $17.8(4.3)$ & $17.4(5.3)$ & $17.3(6.1)$ & $17.5(2.2)$ & $16.1(2.8)$ & $16.1(3.1)$ & $17.6(3.1)$ & $17.0(3.7)$ & $16.0(4.3)$ & $17.0(3.5)$ \\
\hline Group mean & $7.0(7.6)$ & $6.6(7.8)$ & $6.7(7.9)$ & $8.0(6.5)$ & $6.8(6.5)$ & $6.9(6.5)$ & $7.5(7.0)$ & $6.8(7.1)$ & $6.6(6.8)$ & $7.0(7.0)$ \\
\hline
\end{tabular}

ANL: acceptable noise level

was determined by subtracting BNL from MCL (ANL=MCL$\mathrm{BNL}$ ). After the ANL test, participants were asked to judge the degree of familiarity and preference on each music sample using a 4-point scales, as used in the previous study on music familiarity [12]. On this questionnaire, the greater numbers correspond to the higher levels of familiarity or preference. To complete all the task, approximately 90 minutes were needed.

\section{Analysis}

All the statistical analyses were performed using SPSS version 20.0 (IBM Corp., Armonk, NY, USA). First, a 2 (group) $\times 2$ (tempo) $\times 3$ (genre) three-way mixed-model analysis of variance (ANOVA) was conducted on ANLs as dependent variable. Group was a between-subjects variable (low-ANL and high-ANL), and music tempo (fast and slow) and music genre (K-pop, pop, and classical) were used as two withinsubjects variables. Second, in order to examine any relationship of ANLs with subjective responses, non-parametric analyses of Spearman's rank-order correlation were conducted on music preference and music familiarity for low-ANL and high-AL groups, separately. For analyses with a lack of sphericity, the degrees of freedom and $\mathrm{F}$ values were adjusted with the Greenhouse-Geisser correction. If necessary, any post-hoc multiple comparisons were conducted. The critical level of significance was always $p<0.01$ in this study.

\section{Results}

Effect of music tempo and genre on ANLs for low-ANL and high-ANL groups

Table 2 shows the mean ANL and standard deviation for low-ANL and high-ANL groups when background music differed by music tempo and genre. When the background music of slow tempo was presented, the mean ANLs ranged from 2.2 to $2.5 \mathrm{~dB}$ for low-ANL listeners and ranged from 17.3 to 17.8 $\mathrm{dB}$ for high-ANL listeners across three music genres. When the fast-tempo music was presented, the mean ANLs of lowANL group were 4.1, 3.0, and $3.1 \mathrm{~dB}$ and the mean ANLs of 
high-ANL group were 17.5, 16.1, and 16.1 dB for K-pop, pop, and classical music, respectively.

A $2 \times 2 \times 3$ three-way mixed-model ANOVA was performed on ANLs and the results showed that the main effect of group was significant $[F(1,39)=281.5]$. Also, we found that music genre significantly influenced the ANLs $[\mathrm{F}(1.73,67.64)=5.36]$ but music tempo did not significantly influence the ANLs. Results from Bonferroni-adjusted multiple paired-comparisons revealed that the ANLs to K-pop music were significantly greater than the ANLs to pop or classical music. A two-way interaction between tempo and group was significant $[\mathrm{F}(1$, 39) $=9.78]$, showing higher ANLs with fast music for low-ANL group yet in opposite for high-ANL groups. Other two-way interactions as well as a three-way interaction among tempo, genre, and group were not significiant. This indicates that the K-pop music which includes vocal part with Korean lyrics yields the greatest amount of interference as background noise.

\section{Relationship between ANLs and subjective responses (music familiarity and music preference)}

This study investigated individual music familiarity and preference on each music sample in order to examine whether the ANLs would be related to individual's familiarity and preference. The mean values of music familiarity was 2.5 (SD: 0.6) and 2.4 (SD: 0.8) for low-ANL and high-ANL groups, respectively. The mean values of music preference was 2.3 (SD: 0.5 ) and 2.1 (SD: 0.7) for low-ANL and high-ANL groups, respectively.

The independent $t$-test showed that the subjective responses did not statistically differ between low-ANL and high-ANL goups on both music familiarity $[\mathrm{t}(39)=0.37]$ and preference [t(39)=1.01].

Non-parametric analyses of Spearman's rank-order correlation were administered to determine any association between individual noise acceptance and their subjective preference or familiarity to music samples. In order to investigate this, we examined relationship between individual ANLs and subjective responses for low-ANL and high-ANL groups. Table 3 shows the Spearman's coefficients rho for low-ANL and highANL groups. As displayed, for low-ANL individuals, their acceptance of background music was relatively unaffected by music familiarity and preference, regardless of music genre. However, the results on correlated measures revealed a different tendency in high-ANL group. For high-ANL individuals, the ANLs were significantly and negatively related to subjective ratings of music familiarity and preference when the music samples were from K-pop $\left(-0.83<\mathrm{r}_{\mathrm{s}}<-0.73\right)$ or pop genre $\left(-0.87<r_{s}<-0.75\right)$. That is, the subjective preference or familiarity of background noise would affect noise acceptance only for the high-ANL listeners, rather than for all listeners.
Table 3. Spearman's rank-order correlation coefficients rho values between ANLs and subjective familiarity and preference responses across three music genres (K-pop, pop, and classical) for lowANL and high-ANL groups

\begin{tabular}{lcc}
\hline \multicolumn{1}{c}{ Correlated measures } & $\begin{array}{c}\text { Correlation } \\
\text { coefficien } \\
\text { rho }\left(r_{s}\right)\end{array}$ & $\begin{array}{c}\text { Significance } \\
\text { level }(p)\end{array}$ \\
\hline LoW-ANL group & All \\
ANL and familiarity with K-pop & -0.07 & p>0.01 \\
ANL and familiarity with pop & -0.41 & for low-ANL \\
ANL and familiarity with classical & -0.36 & group \\
ANL and preference with K-pop & 0.21 & \\
ANL and preference with pop & -0.50 & \\
ANL and preference with classical & -0.27 & \\
High-ANL group & & \\
ANL and familiarity with K-pop & -0.73 & $p<0.01$ \\
ANL and familiarity with pop & -0.75 & $p<0.01$ \\
ANL and familiarity with classical & -0.54 & $p>0.01$ \\
ANL and preference with K-pop & -0.83 & $p<0.01$ \\
ANL and preference with pop & -0.87 & $p<0.01$ \\
ANL and preference with classical & -0.48 & $p>0.01$ \\
\hline ANL: acceptable noise level & &
\end{tabular}

\section{Discussion}

Although various types of target stories and listening conditions have been used for ANL measure [3-5,10,13], a source of background sound has been limited other than use of multitalker babble noise. Despite a widespread and common use of music in everyday life, little research has explored the effect of music as background noise on ANLs [1,6]. Gordon-Hickey and Moore [6] who used one genre of rock as music samples suggested a need of more research to explore individual susceptibility to background music with controlling musical attributes such as tempo, genre, and instrument composition. This has driven this study to investigate the effect of music tempo and genre on individual ANLs to music.

Results of this study showed that K-pop music was relatively less accepted as background noise, resulting in a relatively greater amont of noise distraction (higher ANL) compared to pop or classical music. Interestingly, the significant effect of genre on ANLs was similar in both low-ANL and high-ANL groups. As one of the possible reasons, the native Korean participants could have been greatly annoyed by K-pop music due to meaningful content of K-pop songs. This seems consistent with previous ANL measures which compared ANLs across five types of competing speech maskers (e.g., 1-male, 1-female, 2-talker, 4-talker, and 8-talker speech masker) [10,14,15]. Considering the features of relatively reduced temporal fluctuations and unintelligible meaning in babble noise as background, researchers have examined individuals' noise acceptance as a 
function of the number of the competing talkers. The findings were congruent such that acceptance of background noise was significantly poorer (or higher ANL) when the background speech masker became more meaningful (i.e., 1- or 2-talker noise) for young normal-hearing listeners [10], adult hearing aid users [14], and adults who use cochlear implant [15]. This indicates that annoyance from background noise may depend on informational masking of noise. Thus, selecting a type of the noise source should be carefully considered for clinical measrues of ANLs and also when comparing data across ANL studies who used different materials.

Although it was not the primary interest of this study, no relation was found between ANLs and individuals' age or hearing threshold, consistent with previous findings [1,2,16,17]. This supports the previous findings that ANL might be an inherent characteristic of individual, which is unchangeable with age or acquired hearing loss of listeners. Recent research revealed that the ANLs could be based on subjective loudness [18], self-control level [19], and personality [20]. Considering the substantial impact of subjective and inherent characteristics of the individuals on their ANLs, it seems important to investigate whether the greater annoyance from K-pop music would be associated with self-rating responses on music familiarity and preference.

To clear this out, the present study explored any association between ANLs and individual familiarity or preference to music samples. The results revealed a different tendency on correlated measures between listeners with low-ANL $(n=29)$ and high-ANL ( $n=12)$. Results showed that the ANLs of low-ANL group were independent of their subjective responses of music familiarity and preference. This seems similar with previous finding [2] who reported no significant correlation between ANLs and individual preference to background noise. GordonHickey and Moore [6] also showed no association between ANLs and participants' music preference. However, we found that the ANLs of high-ANL group depended on music familiarity and preference. Expected result to observe was that the listeners would accept more noise (lower ANL) with less familiar and less preferred background music, meaning a positive association between ANLs and subjective responses (music familiarity and preference). Unexpectedly, negative correlations were observed between ANLs and subjective responses of high-ANL group (less acceptance of background music for less familiar or less preferred music). Possibly, the relatively less familiar or less preferred music could introduce new conflicting cues, resulting in more distraction when following a target story. If a listener had a good strategy to suppress familiar music entirely, then the familiarity of music would be beneficial, as reported using word identification [12]. However, a limitation of the study is the relatively small sample size especially for high-ANL group $(n=12)$ such that more research is needed to determine whether subjective familiarity or preference on noise can affect a person's capacity to accept background noise.

\section{Conclusion}

The purpose of the current study was to evaluate the effects of music genre and tempo on the ANLs to background music. Results showed that, overall, the ANLs did not significantly differ by music tempo. The ANLs depended on the genre of background music. Since K-pop music with more meaningful lyrics was poorly accepted than pop or classical music for both low-ANL and high-ANL groups, the degree of informational masking would decrease acceptance of background noise. The subjective familiarity and preference of noise had a great impact on ANLs only for high-ANL group.

\section{REFERENCES}

1) Nabelek AK, Tucker FM, Letowski TR. Toleration of background noises: relationship with patterns of hearing aid use by elderly persons. J Speech Hear Res 1991;34:679-85.

2) Freyaldenhoven MC, Smiley DF, Muenchen RA, Konrad TN. Acceptable noise level: reliability measures and comparison to preference for background sounds. J Am Acad Audiol 2006;17:640-8.

3) Gordon-Hickey S, Moore RE. Acceptance of noise with intelligible, reversed, and unfamiliar primary discourse. Am J Audiol 2008;17: 129-35.

4) Brännström KJ, Lantz J, Nielsen LH, Olsen SØ. Acceptable noise level with Danish, Swedish, and non-semantic speech materials. Int J Audiol 2012;51:146-56.

5) Ho HC, Wu YH, Hsiao SH, Stangl E, Lentz EJ, Bentler RA. The equivalence of acceptable noise level (ANL) with English, Mandarin, and non-semantic speech: a study across the U.S. and Taiwan. Int J Audiol 2013;52:83-91.

6) Gordon-Hickey S, Moore RE. Influence of music and music preference on acceptable noise levels in listeners with normal hearing. J Am Acad Audiol 2007;18:417-27.

7) Eskridge EN, Galvin JJ 3rd, Aronoff JM, Li T, Fu QJ. Speech perception with music maskers by cochlear implant users and normalhearing listeners. J Speech Lang Hear Res 2012;55:800-10.

8) Başkent D, van Engelshoven S, Galvin JJ 3rd. Susceptibility to interference by music and speech maskers in middle-aged adults. J Acoust Soc Am 2014;135:EL147-53.

9) Hemanth NS, Sankalpa M, Devamma V. The relationship between acceptable noise level and electrophysiologic auditory brainstem and cortical signal to noise ratio. Audiol Res 2014;4:1-4.

10) Shin JB, Lee JH. Effects of the target talker gender and the number of competing talkers on Acceptable Noise Level (ANL) of Korean normal-hearing adults. Audiology 2010;6:146-52.

11) Milliman RE. The influence of background music on the behavior of restaurant patrons. J Consum Res 1986;13:286-9.

12) Russo FA, Pichora-Fuller MK. Tune in or tune out: age-related differences in listening to speech in music. Ear Hear 2008;29:746-60.

13) Gordon-Hickey S, Moore RE, Estis JM. The impact of listening condition on background noise acceptance for young adults with normal hearing. J Speech Lang Hear Res 2012;55:1356-72. 
14) Kim JH, Lee JH, Lee HK. Advantages of binaural amplification to acceptable noise level of directional hearing aid users. Clin Exp Otorhinolaryngol 2014;7:94-101.

15) Baek EJ, Lee JH. Measurement of acceptable noise level and selfreported subjective disability in adult cochlear implant users. Audiology 2012;8:196-203.

16) Nabelek AK, Tampas JW, Burchfield SB. Comparison of speech perception in background noise with acceptance of background noise in aided and unaided conditions. J Speech Lang Hear Res 2004;47: 1001-11.

17) Nabelek AK, Freyaldenhoven MC, Tampas JW, Burchfiel SB,
Muenchen RA. Acceptable noise level as a predictor of hearing aid use. J Am Acad Audiol 2006;17:626-39.

18) Recker KL, McKinney MF, Edwards BW. Loudness as a cue for acceptable noise levels. J Am Acad Audiol 2014;25:605-23.

19) Nichols AC, Gordon-Hickey S. The relationship of locus of control, self-control, and acceptable noise levels for young listeners with normal hearing. Int J Audiol 2012;51:353-9.

20) Franklin C, Johnson LV, White L, Franklin C, Smith-Olinde L. The relationship between personality type and acceptable noise levels: a pilot study. ISRN Otolaryngol 2013;2013:902532. 